\title{
Repetition, cuing, and state-dependent memory
}

\author{
ERIC EICH and ISABEL M. BIRNBAUM \\ University of California, Irvine, California 92717
}

\begin{abstract}
Two experiments examined the effects of several types of repetition on state-dependent memory for conceptually categorized words. In both experiments, compatibility between pharmacological states at encoding and at retrieval facilitated the uncued recall of nonrepeated categories but had no appreciable effect on the recall of words within recalled categories. In both experiments, compatibility between encoding/retrieval states failed to facilitate the uncued recall of repeated categories (i.e., categories whose names and/or exemplars were presented at least twice, in a constant or variable order, and with substantial spacing between successive presentations). And again, in both experiments, the level of uncued recall was higher for repeated than for nonrepeated categories, irrespective of the compatibility or incompatibility of encoding/retrieval states. These findings, together with other relevant observations reported in the literature, suggest that repetition in general, and repetition of category names in particular, influences recall in much the same ways as does explicit cuing with category names: It enhances the accessibility of higher order units, as reflected in uncued category recall, and it diminishes to the extent to which access to these units is state dependent.
\end{abstract}

The role of the pharmacological context or state in which perception and recollection of events take place has long been regarded as potentially important. In his massive treatise, On obscure diseases of the brain .... Forbes Winslow (1860, pp. 338-341) cited several examples of a phenomenon that modern investigators would recognize as dissociative or state-dependent memory, including the case of an Irish porter who forgot, when sober, what he had done while drunk and yet could recapture the memory once he again indulged in "potations pottle deep:" But although the existence of the state-dependence phenomenon was known to early students of memory-Winslow, for instance, among many others (e.g., Luys, 1882; Macnish, 1834; Ribot, 1882)-only within the last decade or so has this knowledge been translated into experimental demonstrations and analytical observations. Consequently, the empirical limits of human state dependence, the circumstances under which the phenomenon occurs and the factors that determine its magnitude, have not yet been firmly fixed. Delineation of these limits is one of the main aims of research on human state-dependent memory, and it is to this end that we focus here on the effects of

Preparation of this paper was facilitated by an intramural grant from the National Institute of Mental Health (via the Laboratory of Clinical Psychopharmacology) and by an extramural grant from the National Institute on Alcohol Abuse and Alcoholism (National Alcohol Research Center Grant AA03506). Richard Stillman was instrumental in the performance of the first experiment, Doris Margolis ably assisted in the second, and Janet Metcalfe offered a cogent critique of both. Our thanks to these people, and also to Arthur Glenberg and Steven Smith for their thoughtful reviews. Requests for reprints should be addressed to the first author at the School of Social Sciences, University of California, Irvine, California 92717. repetition and of explicit retrieval cuing on the occurrence of the state-dependence phenomenon.

Our interest in the relations between repetition, cuing, and state dependence derives from four findings. The first of these has to do with the dissimilar effects of drug-state manipulations on the accessibility of higher order units and of elements within these units. The pertinent evidence comes from an experiment by Eich, Weingartner, Stillman, and Gillin (1975), in which college students studied a categorically structured word list either while under the influence of marijuana or while sober. The list was segmented into several distinct conceptual categories, each consisting of a category name ("precious stones," for example) and a small number of words that fit the category (PEARL, OPAL, and the like). The list was presented to the subjects only once, at a rapid rate, and with the instruction to remember as many words as possible. Several hours later, the subjects were tested for recall of the list words either in the same drug state (marijuana intoxication or sobriety) in which they had studied the list (compatible-state conditions) or in the alternative pharmacological context (incompatible-state conditions). Within each of these conditions, one-half of the subjects were tested for recall in the absence of any explicit reminders (uncued recall) and the remaining subjects were tested in the presence of the category names (cued recall).

Results of the uncued test revealed a strikingly specific form of state-dependent memory. Although the average number of conceptual categories represented in recall was significantly higher in the compatible- than in the incompatible-state conditions of the experiment, the average numbers of words recalled per recalled category were virtually identical under both conditions. The fact 
that experimental variation of the compatibility between study and test drug states had an effect on one measure of uncued recall, but not on the other, suggests that the two measures correspond to different aspects of memory accessibility. One of these (recall of categories) reflects the accessibility of higher order units into which individual word events or elements have been organized, whereas the other (recall of words within recalled categories) represents the accessibility of the elements themselves (Tulving \& Pearlstone, 1966; Tulving \& Psotka, 1971). Cast in these terms, the first finding of interest is this: Under conditions of uncued recall, accessibility of higher order units critically depends upon the compatibility of pharmacological states at study and at test, whereas accessibility of elements within these units is largely independent of the compatibility or incompatibility of study/test states.

The second and third findings of concern relate to results obtained under the cued recall conditions of the Eich et al. (1975) experiment. On one hand, the results provided clear evidence of an overall increase (relative to uncued recall) in the accessibility of higher order units without any appreciable gain in the accessibility of elements within these units. Averaging across compatible and incompatible states, more than twice as many categories were recalled in the presence of the category name cues than were recalled in their absence, whereas roughly equivalent numbers of words per category were recalled in the cued and uncued tests (Eich et al., 1975, Figures 2 and 3). On the other hand, results of the cued test provided no evidence of state dependence with respect to the accessibility of higher order units or of elements within these units. Comparisons between compatible and incompatible states revealed no reliable differences in the cued recall of either categories or words per recalled category. Thus, cuing with category names served not only to enhance the accessibility of higher order units, but also to diminish the extent to which access to these units was state dependent.

The final relevant finding comes from a series of experiments by Mathews and Tulving (1973), whose purpose was to study the effects of several types of repetition in a list-item memory task in which the basic unit of analysis consisted of two words in an explicitly designated conceptual category. Among the types of repetition studied were repetition of the complete basic unit (for instance, "precious stones: PEARL, OPAL. . . [other basic units] . . precious stones: PEARL, OPAL") and repetition of category names only, either before or after presentation of the category exemplars ("precious stones ... precious stones: PEARL, OPAL" as opposed to "precious stones: PEARL, OPAL . . . precious stones"). Relative to a nonrepeated control condition, both types of repetition had a large facilitative effect on nominally noncued word recall-even though, in the case of repetition by category names only, the words themselves were presented only once. The effect was more pronounced when repetitions were spaced rather than massed, and, more important, it manifested itself mainly in increased recall of categories rather than words within recalled categories. Thus it appears that repetition in general, and repetition of category names in particular, affects recall in much the same ways as does explicit cuing with category names: It enhances the accessibility of higher order units, but not that of elements within accessible units (Mathews \& Tulving, 1973).

Granted that both category name repetition and category name cuing have the capacity to facilitate access to higher order units, does repetition share with cuing the capacity to inhibit the occurrence of state dependence? That is, is it the case that repetition of category names, with or without concomitant repetition of category exemplars, acts not only to enhance the accessibility of higher order units, but also to diminish the extent to which access to these units is state dependent? In search of the answer to this question, the following two experiments were performed.

\section{EXPERIMENT 1}

\section{Method}

Design. This experiment explored the effects of two types of spaced repetition on state-dependent memory in a situation in which the basic unit of analysis consisted of a category name and three conceptually related words. We will refer to this basic unit as the $C(W)$ unit, where $C$ signifies the category name and $(W)$ represents the three category exemplars. A list of 12 $\mathrm{C}(\mathrm{W})$ units was presented to every participant in the experiment.

Three conditions of presentation of the list may be distinguished. In one of these conditions, the $12 \mathrm{C}(\mathrm{W})$ units composing the list were presented only once (as in the form "precious stones: PEARL, OPAL, TOPAZ"). In a second condition, the list was presented in its entirety once, and then again, in exactly the same order. Thus, the first and second presentations of one and the same basic unit were separated by the presentation of 11 other basic units (e.g., "precious stones: PEARL, OPAL, TOPAZ. . . [other basic units]. . . precious stones: PEARL, OPAL, TOPAZ"). In the third condition, the C component of every basic unit was presented twice, whereas the (W) component was presented only once. The latter condition consisted of the presentation, before the first complete basic unit, of every category name, accompanied by a silent interval corresponding to the duration of the presentation of three category exemplars (c.g., "precious stones: [silence], [silence], [silence]. . . [other category names and/or complete basic units]... precious stones: PEARL, OPAL, TOPAZ"). The order in which the 12 complete basic units were presented was exactly the same as that in which the 12 category names alone were presented. Collectively, we refer to the three methods of presentation as the study condition factor; individually, we designate the conditions as $C(W), C(W)-C(W)$, and $C-C(W)$. A total of 24 subjects were assigned to the $\mathrm{C}(\mathrm{W})$ study condition, and 12 subjects were assigned to each of the other two conditions.

Every participant in the experiment completed the study session while intoxicated with marjuana. One-half of the subjects assigned to each study condition were subsequently tested for recall of the basic units while in a comparable state of marijuana intoxication; the remaining subjects were tested while sober. In this manner, the three study conditions were 
crossed with two state conditions: compatible (study drug/test drug) and incompatible (study drug/test drug free).

Recall was tested both in the absence of any specific reminders (uncued recall) and in the presence of the category names (cued recall), with the tests given in that order. Subjects completed both tests either in the same pharmacological state in which they had studied the basic units or in the alternative state. Thus, while both study and state conditions were varied between subjects, the two test conditions (uncued and cued recall) were varied within subjects.

Lists. A pool of 24 basic units was created through random sampling of the Battig and Montague (1969) category norms. The three category instances included in each unit were selected at random from among the 3rd to the 12th most common responses to the corresponding category name, as indicated in the norms. The pool was randomly divided to form two lists of 12 basic units ( 36 words) each. Every subject studied and was tested for the recall of both the category names and the category instances composing one of these two lists, with each list represented equally often in every condition of the experiment.

Subjects. Forty-eight men, averaging 23 years of age (range = 21-31 years), participated in the experiment for pay. All of the subjects were students at universities located in or near the District of Columbia, and all were experienced marijuana users, averaging about 4.4 occasions of use per week for the past year. The subjects were screened for serious mental or physical illness by a psychiatrist, and they were asked to refrain from using any psychoactive drugs for $24 \mathrm{~h}$ prior to their arrival at the laboratory.

Procedure. The experiment comprised two sessions, designated study and test, separated by $4 \mathrm{~h}$. All experimental procedures were administered on an individual-subject basis. Table 1 lists the order in which the procedures were carried out, beginning with the initial, baseline assessment of objective and subjective drug effects (explained below).

Every subject was informed, as part of his preliminary instructions, that the purpose of the experiment was to investigate the effects of varying doses of marijuana on memory for meaningfully related words. To this end, he would be asked to study a list that was segmented into 12 distinct conceptual categories, each consisting of a category name and three words related to the category. To make matters clear, two sample categories were presented. The subject also was told that $4 \mathrm{~h}$ after list presentation, he would be asked to recall both the category names and the category exemplars in any order.

Depending on the specific study condition to which he had been randomly assigned, the subject was told that (1) the list would be presented in its entirety just once [Condition C(W)] (2) the entire list would be presented once and then immediately once again in the same order [Condition $\mathrm{C}(W)-\mathrm{C}(W)$ ], or (3) before the list was presented in its entirety, the category names alone would be presented once at a rather leisurely pace [Condition $\mathrm{C}-\mathrm{C}(\mathrm{W})]$.

Following the preliminary instructions, the subject's resting radial pulse was taken for $15 \mathrm{sec}$, and he was then asked to rate his current level of drug intoxication in terms of a 10-point experiential "high" scale $(0=$ not at all high; $9=$ higher than ever before). In this manner, the subject's current level of intoxication could be assessed both objectively (via radial pulse) and subjectively (via experiential high).

Shortly after assessment of baseline objective and subjective effects, the subject was asked to smoke a .7-g cigarette composed of marijuana (obtained from the National Institute of Mental Health, under the aegis of the Psychotomimetic Agents Advisory Committee). Unbeknown to the subject, but with the full knowledge of the experimenter, the marijuana contained $15 \mathrm{mg}$ of $\Delta^{9}$-THC, the psychologically active component of marijuana in humans (Galanter, Wyatt, Lemberger, Weingartner, Vaughan, \& Roth, 1972). The subject was instructed to inhale deeply, hold the breath for $15 \mathrm{sec}$, and repeat this process every $30 \mathrm{sec}$ until the entire cigarette was consumed. Roughly $5 \mathrm{~min}$ were reserved for smoking, and an additional $10 \mathrm{~min}$ (the absorption period) for the drug to take maximum effect (see Galanter et al., 1972).

Following a second assessment of objective and subjective drug effects, list presentation began. Presentation occurred via an audio tape recorder, at a rate of $2.5 \mathrm{sec} /$ category name or exemplar. Thus, in the $\mathrm{C}-\mathrm{C}(\mathrm{W})$ study condition, the initial presentation of every category name was accompanied by a silent interval of $7.5 \mathrm{sec}$ duration. Total presentation time was $2 \mathrm{~min}$ in the $\mathrm{C}(\mathrm{W})$ study condition and $4 \mathrm{~min}$ in the $\mathrm{C}(\mathrm{W})-\mathrm{C}(\mathrm{W})$ and $\mathrm{C}-\mathrm{C}(\mathrm{W})$ conditions.

The subject spent the next several hours in the laboratory listening to music, watching television, or playing cards. Four hours after the initial assessment of objective and subjective drug effects (first baseline), these effects were assessed again (second baseline). The subject was then given a second marijuana cigarette, identical in size, weight, and taste to the first. Depending on the particular state condition (compatible or incompatible) to which the subject had been randomly assigned, the second cigarette contained either $15 \mathrm{mg}$ or (virtually) $0 \mathrm{mg}$ of $\Delta^{\prime}$-THC. Neither the subject nor the experimenter knew in advance whether the second cigarette was or was not a placebo. Pro-

Table 1

Sequence of Procedures (Experiment 1)

\begin{tabular}{cll}
\hline Elapsed Time & \multicolumn{1}{c}{ Procedure } & \multicolumn{1}{c}{ Comment } \\
\hline $0: 00$ & \multicolumn{1}{c}{ Study Session } \\
$0: 05$ & $\begin{array}{l}\text { assess objective/subjective effects } \\
\text { begin smoking (first cigarette) } \\
\text { begin absorption period } \\
\text { assess objective/subjective effects } \\
\text { begin list presentation }\end{array}$ & $\begin{array}{l}\text { first baseline } \\
\text { active marijuana only }\end{array}$ \\
$0: 25$ & \multicolumn{1}{c}{ Test Session } \\
$4: 00$ & assess objective/subjective effects \\
$4: 05$ & begin smoking (second cigarette) & all study conditions \\
$4: 10$ & begin absorption period & second baseline \\
$4: 20$ & assess objective/subjective effects & active or inert marijuana \\
$4: 25$ & begin uncued recall & \\
$4: 30$ & begin cued recall & \\
\hline
\end{tabular}

Note-Elapsed times are expressed as hours:minutes. "Objective effect" refers to radial pulse/15 sec; "subjective effect" refers to experiential high rating. 
cedures involved in the administration of the second cigarette were the same as those involved with the first. Fifteen minutes after administration of the second cigarette, objective and subjective effects were assessed. The test of uncued recall came next. For this purpose, the subject was asked to reproduce, in writing, as many of the previously studied category names and exemplars as possible, in any order. The subject wrote his responses on a form containing 48 numbered lines; the form was reclaimed by the experimenter after $5 \mathrm{~min}$. Next came the test of cued recall. In this situation, the subject was asked to reproduce, in writing, as many of the category exemplars as possible, in any order. Responses were recorded on a form containing the 12 category names, with three numbered lines printed beside each name. Five minutes were allotted for cued recall.

On completion of the cued test, the subject was debriefed and offered a simple meal. Subjects who had received active marijuana in their second cigarette were required to remain in the laboratory for $4 \mathrm{~h}$ following drug administration; those who had received inert marijuana were allowed to leave at their own discretion.

\section{Results and Discussion}

Objective and subjective drug effects. Data reflecting the objective and subjective drug effects of active and inert marijuana were obtained from 27 subjects: 13 in the compatible-state condition and 14 in the incompatiblestate condition. With respect to the objective-effect data, comparison of pulse rates measured (shortly) before and $(15 \mathrm{~min})$ after smoking revealed a mean increase of $27 \%(\mathrm{~N}=27)$ under active marijuana in the study session of the experiment, $24 \%(\mathrm{~N}=13)$ under active marijuana in the test session, and $2 \%(\mathrm{~N}=14)$ under inert marijuana in the test session. With respect to the subjective-effect data, comparison of experiential high ratings taken before and after smoking revealed a mean net increase of 5.17 points $(\mathrm{N}=27)$ under study-session drug, 3.66 points $(\mathrm{N}=13)$ under test-session drug, and 1.54 points $(\mathrm{N}=14)$ under test-session placebo. These data indicate that although the second active marijuana cigarette did not have quite the same "kick" as the first, the administration of active rather than inert marijuana prior to the tests of recall clearly enhanced the compatibility of study and test states.

Analysis of recall. In both this and the next experiment, the focus of attention is on the influence of study and state conditions on test-session uncued recall of higher order units (categories) and of elements within these units (words within recalled categories). Since the data provided by the test of cued recall reflect the combined effects of the prior presentation and of the uncued recall of list items, comparisons of uncued and cued recall data, with the intent of determining the influence of cuing conditions on the occurrence of human state dependence, are of questionable validity (Eich et al., 1975; Mathews \& Tulving, 1973). Consequently, the data given by the cued test will be afforded only cursory consideration.

The criterion for recall of categories was different for the uncued and cued tests of Experiment 1. Since subjects were asked to recall category names as well as category exemplars in the uncued test, recall of category names was used as the index of category accessibility in the uncued test. Since subjects were given the category names in the test of cued recall, category accessibility in the cued test was inferred from the number of categories from which at least one exemplar was recalled.

In both tests, a measure of the recall of words within recallable categories was obtained for each subject by dividing the number of words recalled by the subject by the number of categories for which he was credited as having recalled. Since the methods of scoring category recall were different for the two tests, recall of words within recallable categories could vary from zero to three in the uncued test and from one to three in the cued test.

Using category name recall as the criterion for category recall in the uncued test enables detection of cases in which at least one correct (i.e., previously presented) exemplar of a given category is recalled but the category name is forgotten or of those in which the category name is recalled with no correct exemplars. Such cases were quite uncommon in the present experiment. There were 2 cases in which at least one correct category exemplar was recalled without the correct category name and 10 cases in which the correct category name was recalled without any correct exemplars. These figures may be compared with the 248 cases in which both the correct category name and at least one correct exemplar were recalled. ${ }^{1}$ These results, together with other similar results reported in the literature (e.g., Mathews \& Tulving, 1973), reveal a very high correlation between direct and indirect measures of category recall (i.e., category recall as inferred from the recall of category names vs. category exemplars).

To facilitate comparison of the results of this experiment with those of the next, the recall of both categories and words within recalled categories are expressed as proportions. For each subject, the number of categories recalled was divided by 12 and the number of words recalled per recalled category was divided by 3 . Means of these subject proportions, as well as standard deviations, were derived as summary statistics for each experimental condition and for certain combinations of conditions, as described below.

Uncued recall. Results of this test, in the form of mean proportions of categories and words per category recalled $\left(R_{c}\right.$ and $R_{w / c}$, respectively), appear in Table 2 .

Data on the uncued recall of words within recalled categories were submitted to a three-factor analysis of variance (study condition by state condition by list). Since the exemplars of each category were presented only once in the $C(W)$ and $C-C(W)$ study conditions of the experiment, we may expect the proportions of words recalled per category to be about the same in these two conditions. Indeed, this expectation is in accord with the findings of Mathews and Tulving (1973), 
Table 2

Recall of Categories $\left(R_{c}\right)$ and Words per Category $\left(R_{w / c}\right)$ in the Uncued Test as a Function of Study and State Conditions (Experiment 1)

\begin{tabular}{|c|c|c|c|c|c|c|c|c|}
\hline \multirow{4}{*}{$\begin{array}{c}\text { Study } \\
\text { Condition }\end{array}$} & \multicolumn{8}{|c|}{ State Condition } \\
\hline & \multicolumn{4}{|c|}{$\mathbf{R}_{\mathrm{c}}$} & \multicolumn{4}{|c|}{$\mathrm{R}_{\mathrm{w} / \mathrm{c}}$} \\
\hline & \multicolumn{2}{|c|}{$\begin{array}{l}\text { Com- } \\
\text { patible }\end{array}$} & \multicolumn{2}{|c|}{$\begin{array}{l}\text { Incom- } \\
\text { patible }\end{array}$} & \multicolumn{2}{|c|}{$\begin{array}{l}\text { Com- } \\
\text { patible }\end{array}$} & \multicolumn{2}{|c|}{$\begin{array}{l}\text { Incom- } \\
\text { patible }\end{array}$} \\
\hline & Mean & SD & Mean & SD & Mean & SD & Mean & SD \\
\hline$C(W)$ & .44 & .15 & .31 & .13 & .71 & .15 & .77 & .18 \\
\hline$C-C(W)$ & .67 & .05 & .67 & .09 & .62 & .20 & .71 & .07 \\
\hline$C(W)-C(W)$ & .74 & .23 & .81 & .13 & .91 & .05 & .82 & .14 \\
\hline
\end{tabular}

Note-Study and state conditions are explained in the text.

whose experiments provided a model for the present research. Therefore, in the analysis of the uncued recall of words within recalled categories, the data for the $\mathrm{C}(\mathrm{W})$ and $\mathrm{C}-\mathrm{C}(\mathrm{W})$ study conditions were combined, yielding a grand mean of .68 for the compatible-state condition and .75 for the incompatible-state condition.

Only one significant effect, that of study condition $[F(1,40)=10.86, \eta$ (eta, as per Bruning \& Kintz, $1968)=.46, p<.01]$, emerged from the analysis of the uncued $R_{W / c}$ data. Consistent with results reported by Mathews and Tulving (1973), repetition of the complete basic $C(W)$ unit increased the accessibility of elements within the unit. The absence of a significant effect of state condition and of any interactions involving state (ps $>.05$ ) squares with the observation of Eich et al. (1975) that access to the individual elements of an accessible higher order unit is largely unaffected by manipulations of the compatibility between study/ test states.

Data relevant to the uncued recall of categories were also examined by means of a three-factor analysis of variance, the factors being the same as those used in the analysis of the $R_{W / c}$ data. Since the criterion for category recall in the uncued test was the recall of category names, and since these were presented twice in the $C-C(W)$ and $C(W)-C(W)$ study conditions, no meaningful difference in the levels of uncued category recall would be expected between these two conditions, and no such difference was found by Mathews and Tulving (1973). Therefore, in the analysis of uncued category recall, data provided by the $\mathrm{C}-\mathrm{C}(\mathrm{W})$ and $\mathrm{C}(\mathrm{W})-\mathrm{C}(\mathrm{W})$ conditions were pooled, resulting in grand means of .70 and .74 for the compatible- and incompatible-state conditions, respectively.

The analysis revealed a highly significant effect of study condition $[\mathrm{F}(1,40)=61.16, \eta=.78, \mathrm{p}<.01]$ and a modestly significant interaction of study condition with state $[\mathrm{F}(1,40)=4.12, \eta=.31, \mathrm{p}<.05]$. No other effects, simple or interactive, were significant ( $p s>.05$ ). The finding of a significant effect of study condition replicates Mathews and Tulving's (1973) observation that repetition of category names, with or without concomitant repetition of category exemplars, facilitates access to higher order units. The finding of a significant interaction between study and state conditions suggests that while compatibility between study and test drug states aids the nominally noncued recall of once-presented category names [Condition $\mathrm{C}(\mathrm{W})$ ], it has no substantive effect on the recall of twice-presented category names [Conditions $\mathrm{C}-\mathrm{C}(\mathrm{W})$ and $\mathrm{C}(\mathrm{W})-\mathrm{C}(\mathrm{W})$ ] Together, these findings lead to the conclusion that repetition in general, and repetition of category names in particular, acts not only to enhance the accessibility if higher order units, but also to diminish the extent to which access to these units is state dependent.

Two objections to this conclusion could be raised. First, it is possible to argue that the higher level of nonrepeated category recall observed under the study drug/ test drug condition of the present experiment, relative to the study drug/test drug-free situation, is attributable not to the difference between these two conditions in the compatibility of study/test states, but rather, to a facilitative effect of the drug on test performance. More generally, the argument is that since comparison of performance under the two conditions is confounded by compatibility and test-session differences, it does not provide a suitable basis for drawing conclusions about the occurrence or nonoccurrence of state dependence.

This argument can be easily countered. In several past experiments similar in method to the present one (e.g., Darley \& Tinklenberg, 1974; Eich et al., 1975; Miller, Cornett, Brightwell, McFarland, Drew, \& Wikler, 1977), marijuana has been found to produce either no appreciable effect on the utilization of stored information or a modest impairment, rather than facilitation, of retrieval. (The storage of information, in contrast to its retrieval, has almost always been shown to be sensitive to the depressant effects of the drug; see Miller, 1976.) This being the case, it seems more sensible to attribute the superior performance observed under the study drug/test drug condition of Experiment 1 to the beneficial effect of reinstatement, at retrieval, of the pharmacological context in which nonrepeated categories were encoded, rather than to a drug-produced facilitation of test performance. (An analogous argument and counterargument could be made in relation to Experiment 2, in which alcohol, rather than marijuana, was used to elicit state dependence.)

The second potential objection is more complex than the first, but it too can be readily neutralized. Recall that in the present experiment, study-session procedures were timed so as to allow for list presentation to begin at or near peak drug effect. Thus, to the extent that the subject's state changes or "drifts" (cf. Bower, 1972) in the course of list presentation, it may plausibly be assumed to drift in the direction of increasing sobriety (or, equivalently, decreasing intoxi- 
cation). Also recall that, regardless of the particular study condition to which the subject had been assigned, list presentation began at the same time and, therefore, ended later in the repeated study conditions $[\mathrm{C}-\mathrm{C}(\mathrm{W})$ and $C(W)-C(W)$ ] than in the nonrepeated condition $[C(W)]$. Thus, while single presentation of the categories results in encoding in only one pharmacological statehighly intoxicated-repeated presentation provides an opportunity for encoding the categories in qualitatively different states-highly intoxicated and less highly intoxicated. Now, if it is also assumed that category recall depends, in part, on the compatibility of encoding and retrieval states, the interactive effect of study and state conditions on uncued category recall observed in the present experiment could be interpreted as little more than a procedural artifact. Specifically, while reinstatement of a highly intoxicated state should be critical for the retrieval of nonrepeated categories (since it was only in such a state that these categories were encoded), it should make little difference in the recall of repeated categories whether retrieval occurs in a highly intoxicated state or in a not-so-highly intoxicated state (since, by virtue of the timing of the experimental procedures, it was possible for the subject to encode these categories in qualitatively different states).

To dismiss this artifactual interpretation, it is sufficient to point out that while participants in Experiment 1 may indeed have drifted toward increasing sobriety in the course of the study session, they were by no means as sober at the end of list presentation, repeated or nonrepeated, as they would have been hours later, if tested for recall following the administration of placebo material. In fact, the drift toward sobriety is so slow that it proved necessary to delay recall testing for $4 \mathrm{~h}$ following the administration of active marijuana, in order for the subjects to regain sobriety. Consequently, the critical interaction of study and state conditions observed in the uncued recall of categories in Experiment 1 cannot be plausibly interpreted as a procedural artifact of the kind described above. This conclusion applies even more forcefully to Experiment 2, in which the rate of pharmacological drift was more closely monitored.

Cued recall. Data obtained by this test are summarized in Table 3. Analyses of the cued recall data revealed that, as was true of uncued recall, repetition of category names increased $R_{c}[F(1,40)=4.64, \eta=.32, p<.05]$, whereas repetition of category exemplars increased $\mathrm{R}_{\mathrm{w} / \mathrm{c}} \quad[\mathrm{F}(1,40)=12.19, \eta=.48, \mathrm{p}<.01]$. Also consistent with the results of the uncued test were the findings of nonsignificant simple and interactive effects of state condition on the cued recall of words within categories $\left(F_{S} \leqslant 1.03\right)$. However, in contrast to the results of the uncued test, no significant simple or interactive effects of state condition were obtained in the analysis of the cued recall of categories $\left(F_{s}<1\right)$. More to the point, while compatibility between study
Table 3

Recall of Categories $\left(\mathbf{R}_{\mathbf{c}}\right)$ and Words per Category $\left(\mathbf{R}_{\mathbf{w} / \mathbf{c}}\right)$ in the Cued Test as a Function of Study and State Conditions (Experiment 1)

\begin{tabular}{|c|c|c|c|c|c|c|c|c|}
\hline \multirow{4}{*}{$\begin{array}{c}\text { Study } \\
\text { Condition }\end{array}$} & \multicolumn{8}{|c|}{ State Condition } \\
\hline & \multicolumn{4}{|c|}{$\mathrm{R}_{\mathrm{c}}$} & \multicolumn{4}{|c|}{$\mathrm{R}_{\mathrm{w} / \mathrm{c}}$} \\
\hline & \multicolumn{2}{|c|}{$\begin{array}{l}\text { Com- } \\
\text { patible }\end{array}$} & \multicolumn{2}{|c|}{$\begin{array}{l}\text { Incom- } \\
\text { patible }\end{array}$} & \multicolumn{2}{|c|}{$\begin{array}{c}\text { Com- } \\
\text { patible }\end{array}$} & \multicolumn{2}{|c|}{$\begin{array}{l}\text { Incom- } \\
\text { patible }\end{array}$} \\
\hline & Mean & $\mathrm{SD}$ & Mean & SD & Mean & SD & Mean & SD \\
\hline$C(W)$ & .79 & .18 & .76 & .21 & .66 & .13 & 59 & .17 \\
\hline$C-C(W)$ & .83 & .11 & .88 & .10 & .71 & .16 & .65 & .12 \\
\hline$C(W)-C(W)$ & .92 & .13 & .89 & .11 & .79 & .11 & .84 & .18 \\
\hline
\end{tabular}

Note-Study and state conditions are explained in the text.

and test drug states facilitated the recall of nominally nonrepeated categories in the uncued test [means of .44 and .31 for the compatible- and incompatible-state conditions; $t(22)=2.41, p<.05]$, it had little effect on the cued recall of these categories (means of .79 and .76 for the compatible- and incompatible-state conditions; $t<1)$. Although, at first glance, these results might be seen as evidence for the alleged "cuing effect" in human state dependence (Eich, 1980), the fact that the cued recall data are contaminated, in that they reflect the combined effect of the prior presentation and of the uncued recall of list items, argues for caution in the interpretation of these data.

\section{EXPERIMENT 2}

Although the purpose of Experiment 2, like that of Experiment 1, was to examine the effects of repetition on state-dependent memory for basic categorized units, the methods of the two experiments differed in several respects. Chief among these were (1) the substitution of alcohol for active marijuana as the agent used to produce state dependence, (2) the extension of the interval separating final presentation and final recall of list items from $4 \mathrm{~h}$ to 7 days, and (3) the inclusion in Experiment 2 of a type of repetition not considered in Experiment 1: spaced repetition of basic units in a variable, rather than constant, order.

The origin of our interest in variable-order repetition may be traced most directly to the work of Keane and Lisman (Note 1). As part of their experiment, Keane and Lisman asked a group of college students to study a list composed of eight conceptual categories, each consisting of a category name and four related words. The subjects were presented with the list four times in a different random order each time, and after each presentation they were tested for the nominally noncued free recall of the words. One day later, the subjects were tested twice more for word recall: first in the absence of any explicit reminders, and then in the presence of the category names (uncued and cued recall, 
in our terms). The subjects completed both delayed tests either in the same drug state, alcohol intoxication or sobriety, in which they had studied the categorized list or in the alternative state.

For present purposes, the key finding to emerge from the Keane and Lisman (Note 1) experiment was that compatibility between study and test drug states had a large facilitative effect on the delayed uncued recall of list words. The effect revealed itself more clearly in enhanced accessibility of categories rather than words within accessible categories, and it vanished when category names were provided as aids to recall. Thus, while the results of our first experiment suggest a complete absence of state dependence in the uncued recall of categories presented in a constant order twice, those provided by Keane and Lisman's experiment indicate a robust state-dependent effect in the uncued recall of categories presented in a varying order on four successive occasions. Given these results, it does not seem too unreasonable to think that the order of category repetition might be a critical factor in the occurrence, or nonoccurrence, of state dependence. It was with this thought in mind that our second experiment was planned.

\section{Method}

Design. As in Experiment 1, a list of $12 \mathrm{C}(\mathrm{W})$ units was presented to subjects under one of three study conditions. These involved the presentation of the list, in its entirety, (1) only once [Condition $\mathrm{C}(\mathrm{W})]$, (2) three times, in the same order each time [Condition $C(W)-C(W)]$, or (3) three times, with the units appearing in a different order each time [Condition $\mathrm{C}(\mathrm{W}) \sim \mathrm{C}(\mathrm{W})]$. In the third condition, the constituents of each basic unit (i.e., the category name and exemplars of the category) were presented in the same order on each of the three successive presentation trials; only the order in which the units themselves were presented varied across trials. Thirty-two subjects were assigned to each of the three study conditions.

Immediately following the final -or, in the $\mathrm{C}(\mathrm{W})$ condition, the only-presentation of the list, every subject was tested for the uncued recall of words within the basic units. We designate this test as $\mathrm{UR}_{\mathbf{s}}$, to indicate that it was given at the conclusion of the initial, study session of the experiment.

Every participant completed the study session while intoxicated with alcohol. In the subsequent test session, one-half of the subjects in each study condition were tested for recall whilc in a comparable state of alcohol intoxication (the compatiblestate condition); the remaining subjects were tested while sober (the incompatible-state condition). Recall was tested both in the absence of any discretely identifiable reminders and in the presence of the category names, with the cued test following the uncued. We designate these tests as $U R_{t}$ and $C R_{t}$, to signify that they occurred in the context of the test session of the experiment.

Lists. An aggregate of 24 basic units, each composed of a category name and four related words, was formed through random sampling of the Battig and Montague (1969) norms. The four words included in each basic unit were drawn at random from among the $3 \mathrm{rd}$ to the 12 th most common normative responses to the corresponding category name. The aggregate was randomly divided to yield two lists of 12 basic units (48 words) each. Every subject studied and was tested for the recall of words within one of these two lists. Each list was represented equally of ten in every condition of the experiment.

For purposes of the $\mathrm{C}(W) \sim \mathrm{C}(W)$ study condition, three different sequences of the basic units in each list were prepared. Assignment of units to positions in each sequence was determined randomly, with the proviso that no two units appear in exactly the same order in any two sequences.

Subjects. Ninety-six male students at the University of California at Irvine, averaging 23 years of age (range $=21-32$ years), took part in the project for pay. Prospective participants were screened, via a questionnaire, for serious mental or physical distress. Also by means of a questionnaire, data concerning the nature and frequency of alcohol use were gathered to permit the selection of subjects who were moderate drinkers and who would be comfortable with the dose of alcohol administered in the experiment. The subjects were asked to eat a prescribed breakfast at least $3 \mathrm{~h}$ before each experimental session and to refrain from using alcohol or other psychoactive drugs for $24 \mathrm{~h}$ in advance of their arrival at the laboratory.

Procedure. Subjects were treated individually in both the study and test sessions of the experiment, which were held 7 days apart. The sequence of procedures involved in the experiment, beginning with the initial, baseline assessment of objective and subjective drug effects (described below), is illustrated in Table 4.

Preliminary instructions to the subject included the statement that the purpose of the experiment was to explore the effects of varying doses of alcohol on memory for conceptually related words. To this end, he would be asked to study a list that was divided into 12 distinct conceptual categories, each consisting of a category name and four related words. Two sample categories were presented by way of illustration.

Depending on the particular study condition to which he had been randomly assigned, the subject was told that the list would be presented in its entirety either once [Condition $C(W)$ ] or three times, and if three times, the list would be presented in exactly the same order on every trial [Condition $C(W)-C(W)$ ] or the order of category presentation would vary from one trial to the next [Condition $\mathrm{C}(\mathrm{W}) \sim \mathrm{C}(\mathrm{W})]$. The subject also was informed that immediately following the last (or sole) presentation of the list, he would be asked to recall the category cxemplars in any order and without benefit of any explicit reminders.

Following these instructions, the subject's blood-alcohol level was measured (via an Intoximeter Mark IV gas chromatograph), and he then was asked to rate his current level of drug intoxication in relation to a 4-point experiential "high" scale $(0=$ not at all high; $3=$ highly intoxicated). In this manner, the subject's current level of intoxication could be assessed objectively (via blood-alcohol level) as well as subjectively (via experiential high).

Shortly after assessment of baseline objective and subjective effects, the subject was administered a $1-\mathrm{ml} / \mathrm{kg}$ (body weight) dose of absolute alcohol in the form of two equivalent drinks, each composed of equal volumes of 80-proof vodka and masking solution (lemonade tinctured with peppermint extract). The composition of the drinks was known to the experimenter, but not to the subject. The drinks were served one at a time, over ice, $20 \mathrm{~min}$ apart; the subject was asked to consume each drink at a steady pace within the time allotted. To ensure that list presentation would occur at or near peak drug effect, a 20-min absorption period followed completion of the second drink.

Following a second assessment of objective and subjective drug effects, list presentation began. Presentation occurred via an audio tape recorder at a rate of $2.5 \mathrm{sec}$ per category name or exemplar. Total presentation time was $2.5 \mathrm{~min}$ in the $\mathrm{C}(W)$ study condition and $7.5 \mathrm{~min}$ in the $\mathrm{C}(\mathrm{W})-\mathrm{C}(\mathrm{W})$ and $\mathrm{C}(\mathrm{W}) \sim$ $\mathrm{C}(\mathrm{W})$ conditions.

Immediately following the final (or only) list presentation, the subject was asked to reproduce, in writing, as many of the category exemplars as possible in any order; reproduction of the category names was not specifically requested. Responses were written on a form containing 48 numbered lines; no specific time limit was imposed for the completion of this test (designated $U_{R_{s}}$.

On completion of the $U R_{s}$ test, objective and subjective drug 
Table 4

Sequence of Procedures (Experiment 2)

\begin{tabular}{|c|c|c|}
\hline Elapsed Time & Procedure & Comment \\
\hline \multicolumn{3}{|c|}{ Study Session } \\
\hline $0: 00$ & assess objective/subjective effects & first baseline \\
\hline $0: 05$ & begin first drink & \\
\hline $0: 25$ & begin second drink & alcohol solution only \\
\hline $0: 45$ & begin absorption period & \\
\hline $1: 05$ & assess objective/subjective effects & \\
\hline $1: 10$ & begin list presentation & all study conditions \\
\hline $1: 15^{*}$ & begin uncued recall $\left(\mathrm{UR}_{\mathrm{s}}\right)$ & \\
\hline $1: 20^{*}$ & assess objective/subjective effects & \\
\hline \multicolumn{3}{|c|}{ Test Session } \\
\hline $0: 00$ & assess objective/subjective effects & second baseline \\
\hline $0: 05$ & begin first drink & alcohol or wotar solution \\
\hline $0: 25$ & begin second drink & alcohol or water solution \\
\hline $0: 45$ & begin absorption period & \\
\hline $1: 05$ & assess objective/subjective effects & \\
\hline $1: 10^{*}$ & begin uncued recall $\left(\mathrm{UR}_{\mathrm{t}}\right)$ & \\
\hline $1: 15^{*}$ & begin cued recall $\left(\mathrm{CR}_{\mathrm{t}}\right)$ & \\
\hline
\end{tabular}

Note-Elapsed times are expressed as hours:minutes. "Objective effect" refers to blood-alcohol level; "subjective effect" refers to experiential high rating. Study and test sessions were separated by 7 days. "Approximation.

effects were again assessed. The subject then was engaged for the next $50 \mathrm{~min}$ or so in a variety of nonverbal cognitive tasks. Afterward, he was offered a light lunch and was escorted home when his blood-alcohol level had fallen below $55 \mathrm{mg} \%$ and he no longer showed signs of intoxication.

Seven days later, the subject returned to the laboratory. Shortly after the assessment of baseline objective and subjective effects, he was administered the first of two equivalent drinks. Depending on whether the subject had been randomly assigned to the compatible- or incompatible-state condition of the experiment, each drink contained equal volumes of either masking solution and vodka or masking solution and water. The composition of the drinks was known to neither the experimenter nor the subject. Drinks containing alcohol represented an absolute alcohol dose of $1 \mathrm{ml} / \mathrm{kg}$; glasses containing a mixture of water and masking solution were swabbed with vodka prior to serving. Procedures involved in the delivery of the drinks were the same as those outlined above.

Following a 20-min absorption period, objective and subjective drug effects were assessed. Two tests of free recall of category exemplars that the subject had studied the week before were then given in succession. Both tests were self-paced; neither was anticipated by the subject. Responses on the first test (designated $U R_{t}$ ) were written on a form containing 48 numbered lines; those on the second test (designated $C R_{t}$ ) were written on a form containing the 12 previously presented category names, with four lines, labeled with letters, printed beneath each name.

On completion of the $C R_{t}$ test, the subject participated for about $40 \mathrm{~min}$ in a number of verbal and nonverbal cognitive tasks. He then was provided a simple meal and was debriefed. Subjects who had received alcohol were escorted home once all symptoms of intoxication had subsided; those who had not received alcohol were free to leave after the debriefing.

\section{Results and Discussion}

Objective and subjective drug effects. Uniformly zero readings of blood-alcohol level and of experiential high were obtained prior to the start of both the study and test sessions of the experiment.

With respect to the study session, the administration of alcohol produced a mean blood-alcohol level of
$77 \mathrm{mg} \%(\mathrm{~N}=96)$ shortly before list presentation. By the end of list presentation, mean blood alcohol had fallen by about $4 \%(\mathrm{~N}=32)$ in the nonrepeated $[\mathrm{C}(\mathrm{W})]$ study condition and by about $7 \%(\mathrm{~N}=64)$ in the repeated $[C(W)-C(W)$ and $C(W) \sim C(W)]$ study conditions. A similar slow drift toward sobriety was evident in the data on experiential high. From a prepresentation mean of $2.10(\mathrm{~N}=96)$, experiential high ratings declined by roughly $7 \%(\mathrm{~N}=32)$ in the nonrepeated condition and by about $9 \%(\mathrm{~N}=64)$ in the repeated conditions.

With respect to the test session, subjects who received alcohol $(\mathrm{N}=48)$ generated mean pretest blood alcohol and experiential high ratings of $75 \mathrm{mg} \%$ and 1.90 , respectively, whereas those who received water in lieu of alcohol $(\mathrm{N}=$ 48) experienced no detectable objective effect (bloodalcohol levels were consistently zero) and a very slight subjective effect (mean experiential high rating of .08).

Analysis of recall. The analysis of data in to the two component measures, recall of categories $\left(R_{c}\right)$ and of words within recalled categories $\left(R_{w / c}\right)$, differed slightly from the method used in the first experiment. Since participants in Experiment 2 were not explicitly asked to recall the category names in either of the uncued tests $\left(\mathrm{UR}_{\mathrm{s}}\right.$ or $\left.\mathrm{UR}_{\mathrm{t}}\right)$, an "indirect" measure of category recall-specifically, the recall of at least one of the previously presented exemplars of a given category-was adopted for these two tests. This criterion also was applied to the data generated in the test of cued recall $\left(\mathrm{CR}_{t}\right)$. Recall of words within categories was obtained for each subject by dividing the total number of words recalled in a given test $\left(\mathrm{UR}_{\mathrm{s}}, \mathrm{UR}_{\mathrm{t}}\right.$, or $\left.\mathrm{CR}_{\mathrm{t}}\right)$ by the number of categories recalled in that test. The data for each subject were then converted into simple or unconditionalized proportions using divisors of 12 and 4 in the computation of $R_{c}$ and $R_{w / c}$, respectively. Conditionalized proportions of categories and words per 
category recalled in $\mathrm{UR}_{t}$, given their recall in $\mathrm{UR}_{\mathrm{s}}$, were also computed. The methods used in deriving these conditionalized proportions, and our reasons in doing so, will be explained shortly.

Uncued recall/study session $\left(\mathbf{U R}_{\mathbf{s}}\right)$. Data provided by this test are summarized in the upper portion of Table 5 . Analyses of the $\mathrm{UR}_{\mathrm{S}}$ data indicated that repetition of basic units, in either a constant or variable order, increased the recall of both categories $[F(2,84)=24.12, \eta=.60$, $\mathrm{p}<.01]$ and words per recalled category $[\mathrm{F}(2,84)=$ $13.58, \eta=.49, \mathrm{p}<.01]$. No other effects, simple or interactive, were significant $(\mathrm{ps}>.05)$. Also, while repetition in a constant as opposed to variable order gave somewhat higher mean values of $R_{c}$ and $R_{w / c}$ (when averaged over states), differences between the two types of repetition were not reliable ( $\mathrm{ps}>.05$ ).

Uncued recall/test session $\left(\mathbf{U R}_{\mathrm{t}}\right)$. Results of this test, in the form of unconditionalized proportions of categories and words per category recalled, appear in the central portion of Table 5 .

Analysis of the unconditionalized $R_{c}$ data yielded a mixture of expected and unexpected findings. For instance, while the overall level of category recall was significantly increased by both constant- and variableorder repetition $[\mathrm{F}(2,84)=10.03, \eta=.44, \mathrm{p}<.01]$, it was significantly decreased by compatibility between study and test drug states $[F(1,84)=4.34, \eta=.22$, $\mathrm{p}<.05]$. Also, while the expected significant interaction of study and state conditions was found $[F(2,84)=4.99$, $\eta=.33, \mathrm{p}<.01]$, post hoc analysis of this interaction revealed two unexpected findings: the absence of a significant facilitative effect of study/test compatibility on category recall in the nonrepeated control condition

Table 5

Recall of Categories $\left(R_{e}\right)$ and Words per Category $\left(\mathbf{R}_{\mathbf{w} / c}\right)$ in the Uncued Test as a Function of Study, State, and Test Conditions (Experiment 2)

\begin{tabular}{|c|c|c|c|c|c|c|c|c|}
\hline \multirow{4}{*}{$\begin{array}{c}\text { Study } \\
\text { Condition }\end{array}$} & \multicolumn{8}{|c|}{ State Condition } \\
\hline & \multicolumn{4}{|c|}{$\mathrm{R}_{\mathrm{c}}$} & \multicolumn{4}{|c|}{$\mathbf{R}_{\mathbf{W} / \mathrm{c}}$} \\
\hline & \multicolumn{2}{|c|}{$\begin{array}{l}\text { Com- } \\
\text { patible }\end{array}$} & \multicolumn{2}{|c|}{$\begin{array}{l}\text { Incom- } \\
\text { patible }\end{array}$} & \multicolumn{2}{|c|}{$\begin{array}{l}\text { Com- } \\
\text { patible }\end{array}$} & \multicolumn{2}{|c|}{$\begin{array}{l}\text { Incom- } \\
\text { patible }\end{array}$} \\
\hline & Mean & $\mathrm{SD}$ & Mean & SD & Mean & SD & Mean & SD \\
\hline & \multicolumn{8}{|c|}{$\mathrm{UR}_{\mathrm{S}}$ Test Condition } \\
\hline $\begin{array}{l}\mathrm{C}(\mathrm{W}) \\
\mathrm{C}(\mathrm{W})-\mathrm{C}(\mathrm{W}) \\
\mathrm{C}(\mathrm{W}) \sim \mathrm{C}(\mathrm{W})\end{array}$ & $\begin{array}{l}.44 \\
.68 \\
.63\end{array}$ & $\begin{array}{l}.13 \\
.16 \\
.14\end{array}$ & $\begin{array}{l}.50 \\
.71 \\
.66\end{array}$ & $\begin{array}{l}.14 \\
.11 \\
.12\end{array}$ & $\begin{array}{l}.67 \\
.78 \\
.78\end{array}$ & $\begin{array}{l}.14 \\
.10 \\
.20\end{array}$ & $\begin{array}{l}.62 \\
.85 \\
.77\end{array}$ & $\begin{array}{l}.15 \\
.13 \\
.14\end{array}$ \\
\hline $\mathrm{C}(\mathrm{W}) \sim \mathrm{C}(\mathrm{W})$ & \multicolumn{8}{|c|}{$\mathrm{UR}_{\mathrm{t}}$ Test Condition } \\
\hline $\begin{array}{l}C(W) \\
C(W)-C(W) \\
C(W) \sim C(W)\end{array}$ & $\begin{array}{l}.31 \\
.37 \\
.32\end{array}$ & $\begin{array}{l}.14 \\
.12 \\
.12\end{array}$ & $\begin{array}{l}.25 \\
.44 \\
.47\end{array}$ & $\begin{array}{l}.14 \\
.16 \\
.10\end{array}$ & $\begin{array}{l}.49 \\
.56 \\
.65\end{array}$ & $\begin{array}{l}.14 \\
.13 \\
.11\end{array}$ & $\begin{array}{l}.47 \\
.69 \\
.61\end{array}$ & $\begin{array}{l}.17 \\
.14 \\
.72\end{array}$ \\
\hline$C(W) \sim C(W)$ & \multicolumn{8}{|c|}{$\mathrm{UR}_{\mathbf{t}} \mid \mathrm{UR}_{\mathbf{s}}$ Test Condition } \\
\hline $\begin{array}{l}\mathrm{C}(\mathrm{W}) \\
\mathrm{C}(\mathrm{W})-\mathrm{C}(\mathrm{W}) \\
\mathrm{C}(\mathrm{W}) \sim \mathrm{C}(\mathrm{W})\end{array}$ & $\begin{array}{l}.60 \\
.53 \\
.48\end{array}$ & $\begin{array}{l}.17 \\
.18 \\
.14\end{array}$ & $\begin{array}{l}.42 \\
.58 \\
.69\end{array}$ & $\begin{array}{l}.17 \\
.17 \\
.14\end{array}$ & $\begin{array}{l}.68 \\
.71 \\
.75\end{array}$ & $\begin{array}{l}.16 \\
.17 \\
.13\end{array}$ & $\begin{array}{l}.70 \\
.79 \\
.74\end{array}$ & $\begin{array}{l}.23 \\
.13 \\
.11\end{array}$ \\
\hline
\end{tabular}

Note-Study, state, and test conditions are explained in the text. $[t(30)=1.25, p>.10]$, and the presence of a significant inhibitory effect of study/test compatibility on category recall under conditions of variable-order repetition $[t(30)=3.79, p<.01]$. The only finding from post hoc analysis that turned out as expected was the nonsignificant effect of the compatibility manipulation on category recall under conditions of constant-order repetition $[\mathrm{t}(30)=1.49, \mathrm{p}>.10]$.

Analysis of the unconditionalized $R_{w / c}$ data also yielded a combination of expected and unexpected findings. Although the anticipated significant effect of study condition was observed [with overall levels of word per category recall higher in both of the two repetition conditions than in the nonrepeated condition; $\mathrm{F}(2,84)=13.09, \eta=.49, \mathrm{p}<.01]$, an unanticipated significant interaction between study and state conditions was also observed $[\mathrm{F}(2,84)=3.50, \eta=.28$, $p<.05]$. Post hoc analysis of this interaction revealed that compatibility between study and test states significantly reduced the accessibility of words within categories under conditions of constant-order repetition $[\mathrm{t}(30)=2.71, \mathrm{p}<.05]$ but had no significant influence on the measure in either of the other two study conditions (ts $<1)$.

It is possible that a larger number of expected findings, and a correspondingly smaller number of the unexpected kind, would have obtained had performance in test-session uncued recall been conditionalized on performance in the study-session test. To illustrate, consider the unconditionalized data on category recall in the nonrepeated control condition, summarized in Table 5. Inspection of these data indicates that although compatible-state subjects fared somewhat better than their incompatible-state counterparts in $\mathrm{UR}_{t}$ (mean $R_{c}$ values of .31 and .25 ), they fared somewhat worse in $U_{S}$ (mean $R_{c}$ values of .44 and .50 ). Had test-session uncued recall of nonrepeated categories been conditionalized on study-session uncued recall, it is possible that a significant facilitative effect of study/test compatibility would have been found, as we thought it would. Similarly, had $U R_{t}$ recall of words per category under conditions of constant-order repetition been conditionalized on $\mathrm{UR}_{\mathrm{S}}$ recall, a significant inhibitory effect of study/test compatibility would possibly not have been found, as we thought it would not.

To check these and related possibilities, conditionalized proportions of $R_{c}$ and $R_{w / c}$ were calculated and analyzed. With respect to $R_{c}$, the total number of categories recalled by a given subject in both $U_{t}$ and $\mathrm{UR}_{\mathrm{S}}$ was divided by the total number of categories recalled by the same subject in $U_{S}$. With respect to $\mathbf{R}_{\mathbf{w} / \mathrm{c}}$, the total number of shared words (i.e., words recalled by a given subject in both tests) was divided by the total number of words recalled in $\mathbf{U R}_{\mathbf{S}}$ from shared categories (i.e., categories recalled by the same subject in both tests). In calculating these conditionalized proportions, words and categories that a subject recalled in $U_{t}$ but not in $U$ were necessarily excluded. In 
relative terms, the numbers of words and categories that were excluded on this basis were quite low. Summing over subjects and experimental conditions, a total of 125 words and 30 categories were recalled in $U_{t}$ but not in $\mathrm{UR}_{\mathrm{s}}$. These figures may be compared with the 876 total words and 386 total categories that were recalled in both tests. (Note that the unconditionalized $\mathrm{UR}_{\mathbf{t}}$ data summarized in Table 5 reflect the recall of words and categories that were produced in $\mathrm{UR}_{\mathbf{s}}$ in addition to those that were not.) Apart from their comparative rarity, instances of word and category reminiscence were divided about equally among every combination of study, state, and list conditions.

Once conditionalized values of $R_{c}$ and $R_{w / c}$ were obtained for each subject, means and standard deviations of the subject proportions were calculated. These summary statistics are shown in the lower portion of Table 5, under the heading $\mathrm{UR}_{t} \mid \mathrm{UR}_{\mathrm{S}}$.

Analyses of the conditionalized $R_{c}$ and $R_{w / c}$ data revealed only one significant effect, that being the interaction of study and state conditions on category recall $[F(2,84)=10.91, \eta=.45, p<.01]$. As the conditionalized $R_{c}$ data appearing in Table 5 make plain, only in the nominally nonrepeated $[\mathrm{C}(\mathrm{W})]$ condition did compatibility between study and test drug states increase the likelihood that categories recalled in $\mathrm{UR}_{\mathrm{s}}$ would also be recalled in $\mathrm{UR}_{\mathrm{t}}[\mathrm{t}(30)=2.94$, $\mathrm{p}<.01]$. Our hypothesis, and, by implication, Keane and Lisman's (Note 1) finding, that evidence of state dependence might obtain under conditions of variableorder repetition find no support in the results of the present experiment. In fact, our data indicate a significant inhibitory effect of study/test compatibility on conditionalized category recall in the $\mathrm{C}(\mathrm{W}) \sim \mathrm{C}(\mathrm{W})$ condition $[\mathrm{t}(30)=4.33, \mathrm{p}<.01]$, a finding that we are at a loss to explain. More tractable, perhaps, is the question of why we failed to find evidence of state dependence under conditions of variable-order repetition, whereas Keane and Lisman (Note 1) succeeded. The answer may lie in the simple fact that, whereas in the present study, the effects of repetition on state dependence could be compared with a nonrepeated control condition, the absence of this condition in Keane and Lisman's study necessarily precluded such a comparison. If it is the case that repetition acts to diminish, rather than necessarily eliminate, the extent to which one must rely on pharmacological cues to gain access to higher order units, then it is possible--we think probable-that Keane and Lisman would have found an even larger state-dependent effect in the uncued recall of nonrepeated categories, had they included such categories among their experimental materials. Since a nonrepeated control condition was not represented in the design of the Keane and Lisman experiment, one cannot tell for certain whether the discrepancy between their results and ours, with respect to the presence or absence of state dependence in the uncued recall of categories repeated in a variable order, is more apparent than real.
Table 6

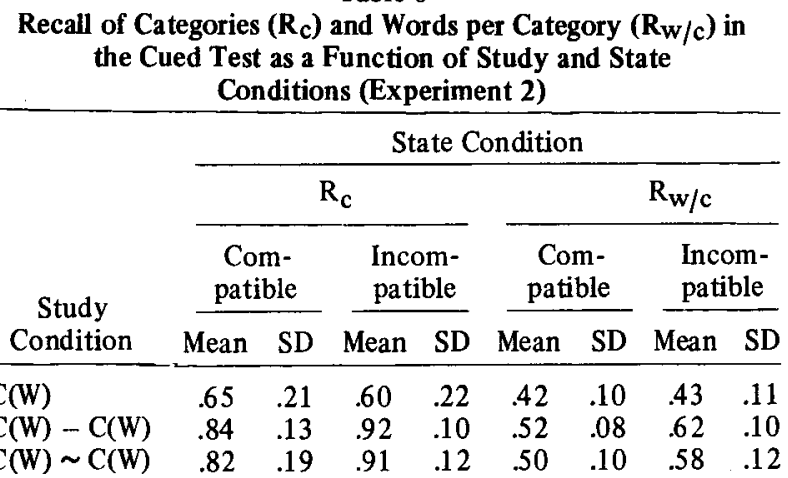

Note-Study and state conditions are explained in the text.

Cued recall/test session $\left(\mathbf{C R}_{\mathbf{t}}\right)$. A summary of the (unconditionalized) data generated in the cued test is given in Table 6 . Analyses of these data revealed a significant facilitative effect of both types of repetition on the cued recall of both categories $[\mathrm{F}(2,84)=24.36$, $\eta=.61, \mathrm{p}<.01]$ and words per category $[\mathrm{F}(2,84)=$ $18.25, \eta=.55, \mathrm{p}<.01]$. Curiously, compatibility between study and test drug states significantly impaired the cued recall of words within categories $[F(1,84)=$ $10.13, \eta=.33, p<.01]$, but the degree of impairment was not correlated with any other experimental variable (ps > .05). Neither the simple effect of state condition nor any interactions involving state was evident in the cued recall of categories ( $\mathrm{ps}>.05$ ).

\section{DISCUSSION}

These two experiments were carried out in search of a general answer to a specific question: Does repetition of category names, with or without concomitant repetition of category exemplars, act not only to enhance the accessibility of higher order units, as reflected in the uncued recall of conceptual categories, but also to diminish the extent to which access to these units is state dependent? The answer given by these experiments is that it does. More to the point, in both of the experiments reported here, compatibility between pharmacological states at study and at test facilitated the uncued recall of nonrepeated conceptual categories but had no appreciable influence on the recall of words within recalled categories. In both experiments, compatibility between study/test states failed to facilitate the uncued recall of repeated categories, that is, categories whose names and/or exemplars were presented at least twice, in a constant or variable order, and with a rather long lag separating successive presentations. And again, in both experiments, the (unconditionalized) level of uncued recall was higher for repeated than for nonrepeated categories, irrespective of the compatibility or incompatibility of study/test states.

That compatibility between study and test drug states aids access to nonrepeated higher order units, but not to elements within these units, is not, of course, a 
novel finding (see Eich et al., 1975), nor is the observation of enhanced higher order unit accessibility following repetition of category names, either alone or in combination with category exemplars (see Mathews \& Tulving, 1973). It is "news," however, to discover that repetition in general, and repetition of category names in particular, serves to diminish the extent to which one must rely on pharmacological cues to gain access to higher order units. This new finding takes on added significance in light of the fact that explicit cuing with category names also serves to inhibit state dependence while it facilitates access to higher order units (Eich et al., 1975). Repetition of and cuing with category names are clearly different experimental operations, in that one has its effect at encoding whereas the other is a factor in retrieval. What these two operations have in common is that both enhance the accessibility of higher order units in a selective manner and both diminish the extent to which access to these units is state dependent. Thus the present findings concerning category repetition, together with earlier observations on category cuing, point to the conclusion that it is the accessibility of higher order units that determines the occurrence or nonoccurrence of state dependence.

Although the conclusion just drawn seems to suit our current and early findings quite well, its generality remains questionable. As part of a series of studies of environmental context and human memory, Smith, Glenberg, and Bjork (1978) performed an experiment in which subjects studied a categorically structured word list in a certain room and were later tested for recall either in that same room or in a different one. Under conditions of uncued recall, compatibility between environmental contexts at study and at test facilitated access not only to the categories, but to words within the categories as well. Clearly, this finding runs counter to the claim that it is only the accessibility of higher order units that is critical for the occurrence of state, or, more broadly considered, context dependence. One potentially important difference between the Smith et al. (1978) experiment and those in which context dependence has been found to be specific to higher order units is the number of exemplars that were included in each category. Whereas experiments that have found context dependence to be localized to the category recall component of total recall have used a small (subspan) number of exemplars per category (typically four or fewer), Smith and his colleagues used a larger (probably superspan) number (eight, to be exact). It is conceivable that when the number of exemplars of a given conceptual category falls within the limits of span, subjects encode the exemplars as individual elements of a single higher order unit, as the experimenter probably intended. However, when the number of exemplars per category exceeds span, the subjects may encode the exemplars as individual elements not of a single experimenter-defined higher order unit, but rather, of many subject-defined higher order units, the exact number and contents of which are probably unknown to the experimenter. In either case, the influence of compatibility or incompatibility of study/test contexts may be localized to the accessibility of higher order units-more specifically, subjective higher order units-rather than to the accessibility of elements within these units. Since techniques exist for identifying subjective units in a variety of situations (e.g., Martin \& Noreen, 1974), it should be possible to experimentally confirm or reject the idea that it is the accessibility of these units that is critical for the occurrence of context dependence. Either way, the exercise is likely to prove stimulating, and perhaps rewarding as well.

\section{REFERENCE NOTE}

1. Keane, T., \& Lisman, S. A. Multiple task disruption of alcohol state-dependent learning. Paper presented at the annual convention of the Eastern Psychological Association, New York, 1976.

\section{REFERENCES}

Battig, W. F., \& Montague, W. E. Category norms for verbal items in 56 categories: A replication and extension of the Connecticut category norms. Journal of Experimental Psychology Monograph, 1969, 80(Whole No. 3, Pt. 2).

Bower, G. H. Stimulus-sampling theory of encoding variability. In A. W. Melton \& E. Martin (Eds.), Coding processes in human memory. Washington, D.C: Winston, 1972.

Bruning, J. L., \& Kintz, B. L. Computational handbook of statistics. Glenview, Ill: Scott, Foresman, 1968.

Darley, C. F., \& Tinklenberg, J. R. Marijuana and memory. In L. L. Miller (Ed.), Marijuana: Effects on human behavior. New York: Academic Press, 1974.

Еich, J. E. The cue-dependent nature of state-dependent retrieval. Memory \& Cognition, 1980, 8, 157-173.

Eich, J. E., Weingartiner, H., Stillman, R. C., \& Gillin, J. C. State-dependent accessibility of retrieval cues in the retention of a categorized list. Journal of Verbal Learning and Verbal Behavior, 1975, 14, 408-417.

Galanter, M., Wyatt, R. J., Lemberger, L., Weingartiner, H., Vaughan, T. B., \& Roth, W. T. Effects on humans of $\Delta^{9}$-tetrahydrocannabinol administered by smoking. Science, 1972, 176, 934-936.

LuYs, J. B. The brain and its functions. New York: Appleton, 1882.

MACNish, R. The philosophy of sleep. New York: Appleton, 1834.

Martin, E., \& Noreen, D. L. Serial learning: Identification of subjective subsequences. Cognitive Psychology, 1974, 6, 421435.

Mathews, R. C., \& Tulving, E. Effects of three types of repetition on cued and noncued recall of words. Journal of Verbal Learning and Verbal Behavior, 1973, 12, 707-721.

Miller, L. L. Marihuana and human cognition: A review of laboratory investigations. In S. Cohen \& R. C. Stillman (Eds.), The therapeutic potential of marihuana. New York: Plenum, 1976.

Minuer, L. L., Cornett, T. L., Brightweli, D. R., McFardand, D. J., Drew, W. G., \& WikLER, A. Marijuana: Effects on storage and retrieval of prose material. Psychopharmacology, $1977,51,311-316$.

Rıвот, T. A. Diseases of memory: An essay in the positive psychology. New York: Appleton, 1882. 
Smith, S. M., Glenbera, A., \& Bjork, R. A. Environmental context and human memory. Memory \& Cognition, 1978, 6, 342-353.

Tulving, E., \& Pearlstone, Z. Availability versus accessibility of information in memory for words. Journal of Verbal Learning and Verbal Behavior, 1966, 5, 381-391.

Tulving, E., \& PsotKa, J. Retroactive inhibition in free recall: Inaccessibility of information available in the memory store. Journal of Experimental Psychology, 1971, 87, 1-8.

WinsLOW, F. On obscure diseases of the brain, and disorders of the mind: Their incipient symptoms, pathology, diagnosis, treatment, and prophylaxis. Philadelphia: Blanchard \& Lea, 1860.

\section{NOTE}

1. Although there were only 2 errors of commission in terms of the uncued recall of category names, there were 58 errors of omission, that is, instances in which the subject correctly recalled one or more exemplars of a given category, yet failed to provide any name for the category. In light of the rarity of commission errors, we think it is likely that the errors of omission reflect noncompliance with the experimental instructions, rather than genuine forgetting of the category names. Accordingly, in the analysis of the uncued recall of categories, subjects who made errors of omission were credited as having recalled the "nameless" categories.

(Received for publication March 27, 1981;

revision accepted November 3,1981 .) 